\title{
Adapted complex structures and Riemannian homogeneous spaces
}

\author{
by RóBerT SzőKe (Budapest)
}

\begin{abstract}
We prove that every compact, normal Riemannian homogeneous manifold admits an adapted complex structure on its entire tangent bundle.

1. Introduction. Adapted complex structures are canonical complex structures on the tangent bundle of Riemannian manifolds. These structures were discovered by investigating certain global solutions of the complex, homogeneous Monge-Ampère equation on Stein manifolds. One can define them in the following way.

We identify the tangent bundle $T \mathbb{R}$ with the complex numbers:

$$
T_{\sigma} \mathbb{R} \ni \tau \frac{\partial}{\partial \sigma} \stackrel{\iota}{\rightarrow} \sigma+i \tau \in \mathbb{C} .
$$

Let now $(M, g)$ be a complete Riemannian manifold and $0<r \leq \infty$. Denote by $T^{r} M$ the domain in $T M$ that consists of vectors having length smaller than $r$. A complex structure on $T^{r} M$ is called adapted if for any geodesic $\gamma: \mathbb{R} \rightarrow M$, the induced map

$$
\gamma_{*}:\left(\gamma_{*}\right)^{-1}\left(T^{r} M\right) \rightarrow T^{r} M
$$

is holomorphic, where $\left(\gamma_{*}\right)^{-1}\left(T^{r} M\right) \subset T \mathbb{R}$ is endowed with the complex structure from (1.1).

The adapted complex structure (if it exists) is unique and when $(M, g)$ is compact and real-analytic and $r$ is small enough, it exists (see [G-S, L-Sz, $\mathrm{Sz}]$ ). The maximal possible radius cannot always be chosen to be infinite. This follows from the fact that the existence of the adapted complex structure on the entire tangent bundle implies that all sectional curvatures are nonnegative.
\end{abstract}

1991 Mathematics Subject Classification: 32M05, 32F07, 32D99, 53C30.

Key words and phrases: adapted complex structures, Riemannian homogeneous spaces.

Research partially supported by the Hungarian Scientific Foundation (OTKA) under grant $\mathrm{T} 21151$. 
So far the only known manifolds with global adapted complex structures were: compact symmetric spaces, a family of surfaces of revolution $[\mathrm{Sz}]$ and a nonstandard metric on $\mathbb{R}^{2}[\mathrm{Sz} 3]$.

The main purpose of this note is to present a simple proof that every compact, normal Riemannian homogeneous manifold admits an adapted complex structure on its entire tangent bundle (Theorem 2.2).

It is very tempting to treat in a similar way all compact Riemannian homogeneous manifolds without the normality condition. But in general such manifolds can have some negative sectional curvatures. We do not know what is the largest possible class of homogeneous manifolds, or say left invariant metrics on a given compact Lie group, that admit global adapted complex structures.

2. Riemannian homogeneous spaces. Throughout this section $K$ will denote a compact Lie group and $L$ a closed subgroup of $K$. Choosing a two sided invariant metric $g$ on $K$ gives rise to the so-called normal metric $g_{n}$ on the homogeneous space $K / L$.

It is well known that $K$ can be imbedded into a unitary group $U(N)$ (for some large $N$ ) and from now on we always tacitly assume this. Denote by $e$ the identity matrix. Following [B-D], the complexified group $K_{\mathbb{C}}$ can be described as follows. The underlying differentiable manifold for $K_{\mathbb{C}}$ is just $K \times \kappa$, where $\kappa=T_{e} K$ is the Lie algebra of $K$. The group structure and the complex structure can be defined by pulling back along the imbedding

$$
\Lambda: K \times \kappa \rightarrow \operatorname{GL}(N, \mathbb{C}), \quad(a, X) \mapsto a \exp (i X) .
$$

For an element $a \in K$ denote by $L_{a}$ the left translation $L_{a}: b \mapsto a b$. With the help of these diffeomorphisms, we can identify $T K$ and $K_{\mathbb{C}}$ :

$$
\Delta: T_{a} K \ni Y \mapsto\left(a,\left(L_{a}\right)_{*}^{-1} Y\right) \in K \times \kappa=K_{\mathbb{C}} .
$$

Denote by $\mathfrak{l}$ the Lie algebra of $L$ and $\mathfrak{m}$ the orthogonal complement, i.e. $\kappa=\mathfrak{l}+\mathfrak{m}$. Via the left $K$-action on itself, the subspace $\mathfrak{m}$ determines a subbundle $\mathfrak{M}$ of $T K$. Define the right $L$-action on $K \times \kappa$ by

$$
(k, X) l:=\left(k l, \operatorname{Ad}\left(l^{-1}\right) X\right) .
$$

Since the metric $g$ is two-sided invariant, the subspace $\mathfrak{m}$ is $\operatorname{Ad}_{L}$ invariant and the quotient space of $K \times \mathfrak{m}$ w.r.t. the $L$-action of $(2.3)$ is the vector bundle $K \times_{L} \mathfrak{m}$.

Let $p: K \rightarrow K / L$ be the projection and $p_{*}$ the induced map $p_{*}: T K \rightarrow$ $T(K / L)$. The derivative of the right $L$-action on $K$ gives a right $L$-action on $\mathfrak{M}$ and the restriction of $\Delta$ to $\mathfrak{M}$ gives an $L$-equivariant identification $\Delta_{\mathfrak{M}}$ between the bundles $\mathfrak{M}$ and $K \times \mathfrak{m}$. The quotient space $\mathfrak{M} / L$ is precisely $T(K / L)$ and the quotient map is just $p_{*}$. The map $\Delta_{\mathfrak{M}}$ descends to a map 
$\Delta_{K / L}: T(K / L) \rightarrow K \times_{L} \mathfrak{m}$ and the following diagram commutes:

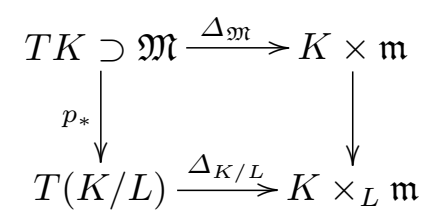

The quotient map $p:(K, g) \rightarrow\left(K / L, g_{n}\right)$ is a Riemannian submersion and at any point $k \in K$ the horizontal subspace is precisely $\mathfrak{M}_{k}$.

After all these preliminaries we only need to recall a result of Mostow (see $[\mathrm{M}])$.

TheOrem 2.1. The map

$$
\Phi: K \times_{L} \mathfrak{m} \rightarrow K_{\mathbb{C}} / L_{\mathbb{C}}, \quad(k, v) \mapsto k \exp (i v) L_{\mathbb{C}}
$$

is a K-equivariant diffeomorphism.

Now we are ready to prove:

THEOREM 2.2. Let $K$ be a compact Lie group and $L$ a closed subgroup of $K$. Let $g$ be a two-sided invariant Riemannian metric on $K$. Then $\Phi \circ \Delta_{K / L}$ is a biholomorphism between $T(K / L)$ (with the complex structure adapted to $\left.g_{n}\right)$ and $K_{\mathbb{C}} / L_{\mathbb{C}}$.

Proof. Step 1. Suppose $L=\{e\}$. We need to show that pulling back the complex structure of $\operatorname{GL}(N, \mathbb{C})$ by $\Lambda \circ \Delta: T K \rightarrow K \times \kappa \rightarrow \operatorname{GL}(N, \mathbb{C})$ is adapted to $g$. Let $\gamma$ be an arbitrary geodesic through $b \in K$. Since $g$ is two-sided invariant, $\gamma$ can be written as $\gamma(\sigma)=b \exp (\sigma X)$ for some $X \in \kappa$. Since for any $\sigma+i \tau \in \mathbb{C}$,

$$
\begin{aligned}
\left(L_{\gamma(\sigma)}\right)_{*}^{-1}(\tau \dot{\gamma}(\sigma)) & =\left.\tau \frac{d}{d t}\right|_{t=\sigma}\left[\gamma(\sigma)^{-1} \gamma(t)\right] \\
& =\left.\tau \frac{d}{d t}\right|_{t=\sigma}\{\exp [(t-\sigma) X]\}=\tau X
\end{aligned}
$$

the composition map $\Lambda \circ \Delta \circ \gamma_{*}: \mathbb{C} \rightarrow \mathrm{GL}(N, \mathbb{C})$ is

$$
\zeta:=\sigma+i \tau \mapsto b \exp (\sigma X) \exp (i \tau X)=b \exp (\zeta X),
$$

that is holomorphic in $\zeta$. Hence the complex structure is indeed adapted.

Step 2. Let now $\gamma: \mathbb{R} \rightarrow K / L$ be a $g_{n}$ geodesic. Since the projection $\operatorname{map} p:(K, g) \rightarrow\left(K / L, g_{n}\right)$ is a Riemannian submersion, $\gamma$ can be lifted to a horizontal geodesic $\widetilde{\gamma}: \mathbb{R} \rightarrow K$ (see for example [Be, p. 245, 9.42]). Horizontal means that in fact $\widetilde{\gamma}_{*}: T \mathbb{R} \rightarrow \mathfrak{M}$, i.e. $\widetilde{\gamma}(\sigma)=b \exp \sigma X$, where $X \in \mathfrak{m}$.

To show that the complex structure on $T(K / L)$ given by the pull-back w.r.t. $\Phi \circ \Delta_{K / L}$ is adapted to $g_{n}$, it is necessary and sufficient to check that the composition $\Phi \circ \Delta_{K / L} \circ \gamma_{*}: T \mathbb{R} \rightarrow K_{\mathbb{C}} / L_{\mathbb{C}}$ is holomorphic. This follows 
from Step 1, the discussion before Theorem 2.1, the holomorphicity of $\pi$ and the commutativity of the diagram below. (We think of the group $K_{\mathbb{C}}$ as a complex subgroup of $\mathrm{GL}(N, \mathbb{C}), K_{\mathbb{C}}=\Lambda(K \times \mathfrak{m})$.)

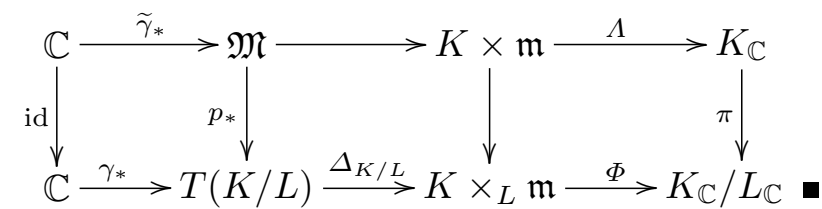

REMARKS. Theorem 2.2 was proved, using different methods, by M. Stenzel in an unpublished preprint in the special case when $K$ is semisimple.

The norm-square function is a strictly plurisubharmonic exhaustion (see [L-Sz]) function on the complex manifold $\left(T(K / L), J_{A}\right)=X$. Consequently, $X$ is Stein. This gives another proof of an old result of Matsushima [Ma], that the complex homogeneous manifold $K_{\mathbb{C}} / L_{\mathbb{C}}$ is Stein.

The uniqueness of the adapted complex structure together with its very definition imply that any isometry $\varphi:(M, g) \rightarrow(M, g)$ induces a biholomorphism $\varphi_{*}$ of the tube $T^{r} M$ (where the adapted complex structure is defined). Assume now that $r$ is finite. It is a natural question to ask whether these are the only biholomorphisms or not. In this generality practically nothing is known, not even in the particular case when $M$ is the real hyperbolic space.

If we assume that $M$ is compact, the answer is almost complete. Namely it is known that $\operatorname{Aut}\left(T^{r} M\right)$ is a compact Lie group [Sz2] and the identity component $\operatorname{Aut}_{0}\left(T^{r} M\right)$ coincides with the identity component of the isometry group $[\mathrm{Bu}]$.

An interesting particular case is when $(M, g)$ is a compact hyperbolic manifold of constant sectional curvature $=-1$. Then the maximal tube where an adapted complex structure exists has radius $\pi / 2$. It was shown by Kan [Ka2] that there is precisely one radius, namely $r=\pi / 4$, such that the hypersurface $S_{r}=\left\{\|\|_{g}=r\right\} \subset T^{\pi / 2} M$ is spherical. She also proved that there exists an $\varepsilon>0$ such that for any $s \in(\pi / 4-\varepsilon, \pi / 4+\varepsilon)$, $\operatorname{Aut}\left(T^{s} M\right)=\operatorname{Isom}(M, g)$.

A closely related question was considered by Burns [Bu]. He showed that the complex manifold structure of the tube $T^{r} M$ completely determines the manifold $M$ and the metric. More precisely, he proved the following. Suppose $(M, g)$ and $(N, h)$ are compact Riemannian manifolds with adapted complex structures on $T^{r} M$ and $T^{r} N(0<r<\infty)$. Assume that $T^{r} M$ and $T^{r} N$ are biholomorphic. Then $(M, g)$ and $(N, h)$ are isometric.

Our observation is based on this theorem.

Proposition 2.3. Let $\left(M^{n}, g\right)$ be a compact Riemannian manifold, $n>1$ and $0<R \leq \infty$. Suppose $T^{R} M$ admits an adapted complex struc- 
ture. Then for any $0<r<s<R$, the hypersurfaces $S_{r}:=\left\{\|\|_{g}=r\right\}$ and $S_{s}:=\left\{\|\|_{g}=s\right\}$ are not $C R$-equivalent.

Pro of. Suppose there exists a CR-diffeomorphism $\Phi: S_{r} \rightarrow S_{s}$. Then $\Phi$ extends to a biholomorphism $\widetilde{\Phi}: T^{r} M \rightarrow T^{s} M$. Let $\lambda=r^{2} / s^{2}$ and $h:=\lambda g$. Then the adapted complex structure w.r.t. $h$ is the same as for $g$, and the tube $T^{s} M$ w.r.t. $g$ is the same as the tube with radius $r$ w.r.t. $h$.

Thus the above mentioned result of Burns implies the existence of an isometry $\varphi:(M, g) \rightarrow(M, \lambda g)$. This yields $\lambda=1$, a contradiction.

If the compactness condition is dropped, nothing is known. In particular it is not known whether in the case of the real hyperbolic space tubes (with the adapted complex structure) with different radii can be biholomorphic or not.

Now we discuss the symmetries (i.e. biholomorphisms) of the complex manifold $\left(T(K / L), J_{A}\right)$. For further information concerning symmetries of tubes $T^{r} M$ in general, see [Bu, Ka, Sz2].

Proposition 2.4. Let $f: K_{\mathbb{C}} / L_{\mathbb{C}} \rightarrow L_{\mathbb{C}}$ be holomorphic, and $\pi: K_{\mathbb{C}} \rightarrow$ $K_{\mathbb{C}} / L_{\mathbb{C}}$ be the projection map. Then the map $\Phi_{f}: K_{\mathbb{C}} \rightarrow K_{\mathbb{C}}$ defined by

$$
a \mapsto a f(\pi a)
$$

is a biholomorphism of $K_{\mathbb{C}}$.

Proof. It is clear that $\Phi_{f}$ is holomorphic. Suppose

$$
a f(\pi a)=b f(\pi b)
$$

Then $\pi(a f(\pi a))=\pi(b f(\pi b))$, hence $\pi(a)=\pi(b)$. This together with (2.4) implies $a=b$, i.e. $\Phi_{f}$ is injective.

Let now $b \in K_{\mathbb{C}}$. Define $a \in K_{\mathbb{C}}$ by

$$
a:=b[f(\pi(b))]^{-1} .
$$

It is easy to see that $\Phi_{f}(a)=b$, thus $\Phi_{f}$ is onto.

REMARK. It is also obvious that $\Phi_{f} \equiv \Phi_{g}$ iff $f \equiv g$.

Corollary 2.5. Aut $\left(K_{\mathbb{C}}\right)$ is at least as large as the set of holomorphic maps

$$
\operatorname{Hol}\left(K_{\mathbb{C}} / L_{\mathbb{C}} \rightarrow L_{\mathbb{C}}\right) .
$$

Corollary 2.6. If $K \neq S^{1}$, then $\operatorname{Aut}\left(K_{\mathbb{C}}\right)$ is infinite-dimensional.

Pro of. If $K$ is not a torus, take for instance $L$ to be a maximal torus in $K$. Since both $K_{\mathbb{C}} / L_{\mathbb{C}}$ and $L_{\mathbb{C}}$ are Stein, the statement follows from Corollary 2.5. If $K$ is a torus, but not the circle, $K_{\mathbb{C}}$ can be identified with $\left(\mathbb{C}^{*}\right)^{n}, n>1$. Now if $h$ is any holomorphic function on $\mathbb{C}$, the map $\Phi_{h}:\left(\mathbb{C}^{*}\right)^{n} \rightarrow\left(\mathbb{C}^{*}\right)^{n}$, is an element of $\operatorname{Aut}\left(\mathbb{C}^{*}\right)^{n}$.

$$
\Phi_{h}\left(z_{1}, \ldots, z_{n}\right)=\left(e^{h\left(z_{1} z_{2}\right)} z_{1}, e^{-h\left(z_{1} z_{2}\right)} z_{2}, z_{3}, \ldots, z_{n}\right),
$$


Acknowledgements. The author thanks the Max Planck Institut für Mathematik, Bonn and The Banach Center, Warsaw for their support.

\section{References}

[B-D] T. Bröcker and T. tom Dieck, Representations of Compact Lie Groups, Springer, New York, 1985.

[Be] A. L. Besse, Einstein Manifolds, Springer, 1987.

[Bu] D. Burns, On the uniqueness and characterization of Grauert tubes, in: Complex Analysis and Geometry, V. Ancona, E. Ballico and A. Silva (eds.), Marcel Dekker, 1996, 119-133.

[G-S] V. Guillemin and M. Stenzel, Grauert tubes and the homogeneous MongeAmpère equation I, J. Differential Geom. 34 (1991), 561-570.

[Ka] S. J. Kan, On the rigidity of non-positively curved Grauert tubes, preprint, 1996.

[Ka2] -, The asymptotic expansion of a CR invariant and Grauert tubes, Math. Ann. 304, (1996), 63-92.

[L-Sz] L. Lempert and R. Szöke, Global solutions of the homogeneous complex MongeAmpère equation and complex structures on the tangent bundle of Riemannian manifolds, Math. Ann. 291 (1991), 689-712.

[Ma] Y. Matsushima, Espaces homogènes de Stein des groupes de Lie complexes, Nagoya Math. J. 16 (1960), 205-218.

[M] G. D. Mostow, Some new decomposition theorems for semi-simple groups, Mem. Amer. Math. Soc. 4 (1955), 31-54.

[Sz] R. Szőke, Complex structures on the tangent bundle of Riemannian manifolds, Math. Ann. 291 (1991), 409-428.

[Sz2] - Automorphisms of certain Stein manifolds, Math. Z. 219 (1995), 357-385.

[Sz3] - Adapted complex structures on tangent bundles of Riemannian manifolds, in: Complex Analysis and Generalized Functions (Varna, 1991), I. Dimovski and V. Hristov (eds.), Publ. House Bulgarian Acad. Sci., Sofia, 1993, 304-314.

Department of Analysis

Eötvös University

Múzeum krt. 6-8

1088 Budapest, Hungary

E-mail: rszoke@cs.elte.hu 